\title{
Radio Wave Propagation Characteristics in FMCW Radar
}

\section{Ghada M. SAMI}

Mathematics Department, Faculty of Science, Ain Shams University, Cairo, Egypt

Email: g_sami2003@yahoo.com

Received July $3^{\text {rd }}, 2009$; revised August $11^{\text {th }}, 2009$; accepted August $20^{\text {th }}, 2009$.

\begin{abstract}
FMCW Radar (Frequency Modulated Continuous Wave Radar) is used for various purposes, such as atmospheric Remote Sensing, inter-vehicle ranging, etc. FMCW radar systems are usually very compact, relatively cheap in purchase as well as in daily use, and consume little power. In this paper, FMCW radar determines a target range by measuring the beat frequency between a transmitted signal and the received signal from the target, and Combines between PO and radar single. The approach based on frequency domain physical optics for the scattering estimation and the linear system modeling for the estimation of time domain response, and FMCW Radar signal processing.
\end{abstract}

Keywords: Radio Wave, FMCW Radar, Cloud Profiling Radar

\section{Introduction}

The FMCW radar has to adjust the range of frequencies of operation to suit the material and targets under investigation. The transceiver generates a signal of linearly increasing frequency for the frequency-sweep period. The signal propagates from the antenna to a static target and back. The value of the received-signal frequency compared to the transmitted-signal frequency is proportional to the propagation range. The main advantages of the FMCW radar are the wider dynamic range, lower noise figure and higher mean powers that can be radiated. In addition a much wider class of antenna is available for use by the designer.

The further advantage of FMCW radar is its ability to adjust the range of frequencies of operation to suit the material and targets under investigation if the antenna has an adequate pass-band of frequencies. This radar system mixes the wave reflected by a target object and part of the radiated wave to obtain a beat signal that contains distance and speed components. For large scatterer, the physical-optics approximation is an efficient method in the frequency domain $[1,2]$. This physical optics (PO) approximation is initially applied in the frequency-domain with the inverse Fourier transform [3], [4], [5], [6]. With FMCW, the high-frequency circuitry for beat signal detection is relatively simple and distance can be directly obtained. By mixing the received FMCW and transmitted FMCW signals, the system obtains a beat signal having a frequency $f_{b}$.

\section{The Principles of the FMCW Radar}

The principle of the FMCW radar is shown in Figure 1. Transmitted signal from one of the antennas is reflected, and is received by the other antenna with delay time $T_{o}$ relative to the original transmitted signal. Mixing the received and transmitted frequencies, the beat frequencies $f_{b}$ are observed in the spectra.

The time takes for the signal to travel the two-way distance between the target and the radar is $T_{o}$, hence [7], [8]:

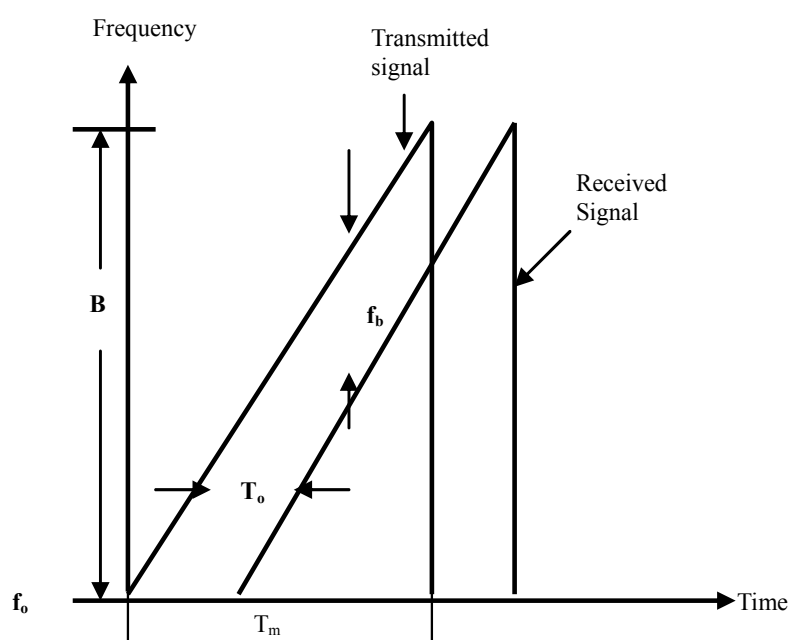

Figure 1. Principle of the FMCW radar 


$$
T_{o}=\frac{2 R}{c}
$$

From the geometry of transmit and receive waveforms we can derive a relationship between the beat frequency $f$ $b$, the range $R$, and $c$ is the velocity of light.

From Figure 1, we can see,

$$
\frac{T_{o}}{T_{m}}=\frac{f_{b}}{B}
$$

Substituting (1) in (2), we get

$$
f_{b}=\frac{2 R B}{c T_{m}}
$$

\section{Scattered Wave from Radar Target}

\subsection{Frequency Domain Physical Optics}

For a perfectly conducting body, the frequency-domain PO-induced current distribution over the illuminated surface is $[9,10,11]$ :

$$
\vec{j}_{s}^{p o}(\vec{R}, \omega)=2 \hat{n} \times \vec{H}^{i n c}(\vec{R}, \omega)
$$

where $\hat{n}$ is the unit vector normal to the surface $\bar{R}$ and $\bar{H}^{i n c}(\bar{R}, \omega)$ is the incident magnetic field with angular frequency $\omega$.

The frequency-domain scattered field is obtained by calculating the integral over the illuminated surface using

$$
H(\omega)=\frac{\eta_{o}}{4 \pi F_{c}(\omega) V_{i}(\omega)} \sum_{k} \frac{1}{\left|\bar{R}-\bar{R}_{k}\right|}
$$

where $F_{c}(\omega)$ is the Complex antenna factor.

\subsection{Treatment of FMCW Signal}

Instantaneous frequency $f_{i}(t)$, is given as

$$
f_{i}(t)=\left(f_{o}+\frac{t}{T_{m}} B\right) \quad\left(0 \leq t \leq T_{m}\right)
$$

and the instantaneous phase $\varphi_{i}(t)$, is defined as

$$
\varphi_{i}(t)=2 \pi \int_{0}^{t} f_{i}(\tau) \mathrm{d} \tau=2 \pi f_{o} t+\pi \frac{B}{T_{m}} t^{2}
$$

Using Equation (9), the FMCW signal waveform is defined as

$$
v_{i}(t)=V_{i} \operatorname{Cos}\left(2 \pi f_{o} t+\pi \frac{B}{T_{m}} t^{2}\right)
$$

The output of mixer $\mathrm{V}_{\mathrm{FMCW} 1}(\mathrm{t})$ is expressed as

$$
\mathrm{v}_{\mathrm{FMCW1}}(t)=v_{\mathrm{i}}(\mathrm{t}) v_{o}(t)
$$

The output waveform $v_{o}(t)$ is

$$
v_{o}(t)=v_{i}(t) * h(t)
$$

when $h(t)$ is a sample delay of $T_{o}$, i.e. the free space Green's function:

$$
\bar{H}^{i n c}(\bar{R}, \omega) \bar{E}^{P O}(\bar{R}, \omega)=-\eta_{o} \iint_{S} \bar{j}_{s t}^{P O}\left(\bar{R}^{\prime}, \omega\right) \frac{e^{-j k_{o}\left|\bar{R}-\bar{R}^{\prime}\right|}}{4 \pi|\bar{R}-\bar{R}|} \mathrm{ds}{ }^{\prime}
$$

where the vector $\bar{R}$ locates the integration point on the scatterer surface, $k_{o}$ is the wave number, $c=\frac{2 \pi f}{k_{o}}$ is the velocity of the light and $\eta_{o}$ is the intrinsic free space impedance, and $\vec{j}_{s t}^{p o}(\bar{R}, \omega)$ is the surface-current distribution.

The frequency transfer function $H(\omega)$ is defined as

$$
H(\omega)=\frac{V_{o}(\omega)}{V_{i}(\omega)}
$$

where $V_{i}(\omega)$ is the input waveform in frequency domain physical optics, this is just a magnitude of the source.

The output Voltage $V_{o}(\omega)$ is calculated from $E^{p o}(R, \omega)$ by considering the receiver antennas as $[12,13]$,

$$
V_{o}(\omega)=\frac{E^{p o}(R, \omega)}{F_{c}(\omega)}
$$

$\left\{2 \hat{\mathrm{n}} \times \overline{\mathrm{H}}^{\mathrm{inc}}\left(\bar{R}_{k}, \omega\right)\right\} e^{-j k_{o}\left|\bar{R}-\bar{R}_{k}\right|} \Delta \mathrm{s}_{\mathrm{k}}$

Therefore, mixer output signal is

where

$$
\mathrm{v}_{\mathrm{FMCW} 1}(t)=v_{i}(t) v_{i}\left(t-T_{o}\right)
$$

$v_{i}\left(t-T_{o}\right)=V_{i} \operatorname{Cos}\left[2 \pi f_{o}\left(t-T_{o}\right)+\pi \frac{B}{T_{m}}\left(t-T_{o}\right)^{2}\right]$

From Equations (10) and (11) we can calculate:

$$
\begin{aligned}
& V_{F M C W 1}(t)=\frac{V_{i}^{2}}{2}\left\{\operatorname{Cos}\left(4 \pi f_{0} t-2 \pi f_{o} T_{o}+\pi \frac{B}{T_{m}}\left(2 t^{2}-2 T_{0} t+T_{0}^{2}\right)\right)\right. \\
& \left.+\operatorname{Cos}\left(2 \pi f_{o} T_{o}+\pi \frac{B}{T_{m}}\left(2 T_{o} t-T_{o}^{2}\right)\right)\right\}
\end{aligned}
$$

The first Cos term describes a linearly increasing FM signal (chirp) at about twice the carrier frequency with a phase shift that is proportional to the delay time $T_{0}$. This term is generally filtered out.

The second Cos term:

$$
V_{F M C W 2}(t)=\frac{V_{i}^{2}}{2} \operatorname{Cos}\left(2 \pi f_{o} T_{o}+\pi \frac{B}{T_{m}}\left(2 T_{o} t-T_{o}^{2}\right)\right)
$$


describes a beat signal at a fixed frequency $f_{b}=\frac{B}{T_{m}} T_{o}$.

\subsection{Combination of PO and Radar Single Processing}

As $h(t)$ given in section 3.2 is just an idealized model, more realistic $h(t)$ obtained by $\mathrm{PO}$ in section 3.1, Equation (12) shall be used.

However, Equation (6) is given in frequency domain $H(\omega)$, and it shall be Inverse Fourier transformed

$$
h(t)=\frac{1}{2 \pi} \int_{-\infty}^{\infty} H(\omega) \mathrm{e}^{\mathrm{j} \omega \mathrm{t}} \mathrm{d} \omega
$$

It is sufficient that $H(\omega)$ is computed only within the source frequency range, i.e. $f_{o} \leq f \leq f_{o}+B$. Outside the band, $H(\omega)$ can be assumed zero, $V_{i}(\omega)$ is also zero in this region.

In reality, Fourier transform shall be executed numerically. Let us assume the sampling interval $\Delta T$ which shall satisfy the following relation

$$
\begin{gathered}
\Delta T \leq \frac{1}{2\left(f_{o}+B\right)} \\
f_{s}=\frac{1}{\Delta T} \\
f_{o}=N_{L} f_{s} \\
f_{o}+B \leq\left(N_{L}+N_{B}\right) f_{s}
\end{gathered}
$$

where $f_{s}$ is a sampling frequency, $N_{L}$, and $N_{B}$ are some certain integers.

Now $v_{i}(t)$ is denoted as $v_{i}(l \Delta T)$, and $h(n \Delta T)$ is given as,

$$
\begin{aligned}
& h(n \Delta T)=\frac{f_{s}}{4\left(N_{L}+N_{B}\right)}\left(\sum _ { m = N _ { L } } ^ { N _ { L } + N _ { B } } \left(H\left(m 2 \pi \mathrm{f}_{\mathrm{s}}\right) \mathrm{e}^{\mathrm{j} \mathrm{m} 2 \pi f_{s} n \Delta T}\right.\right. \\
& \left.\left.+H^{*}\left(\mathrm{~m} 2 \pi f_{s}\right) \mathrm{e}^{-\mathrm{j} \mathrm{m} 2 \pi f_{s} n \Delta T}\right)\right)
\end{aligned}
$$

Convolution (11) is now implemented as,

$$
v_{o}(l \Delta T)=\Delta T \sum_{n=0}^{2\left(N_{L}+N_{B}\right)} v_{i}((l-n) \Delta T) \mathrm{h}(\mathrm{n} \Delta T)
$$

Substituting from (23) and (10) in (13), we can get

$$
\begin{gathered}
V_{\text {fncwl }}(t)=V_{i}^{2} \operatorname{Cos}\left(2 \pi f_{o} t+\pi \frac{B}{T_{m}} t^{2}\right) \\
\Delta T \sum_{n=0}^{2\left(N_{L}+N_{B}\right)} \operatorname{Cos}\left(2 \pi f_{o}((l-n) \Delta T)+\pi \frac{B}{T_{m}}((l-n) \Delta T)^{2}\right) \mathrm{h}(\mathrm{n} \Delta T)
\end{gathered}
$$

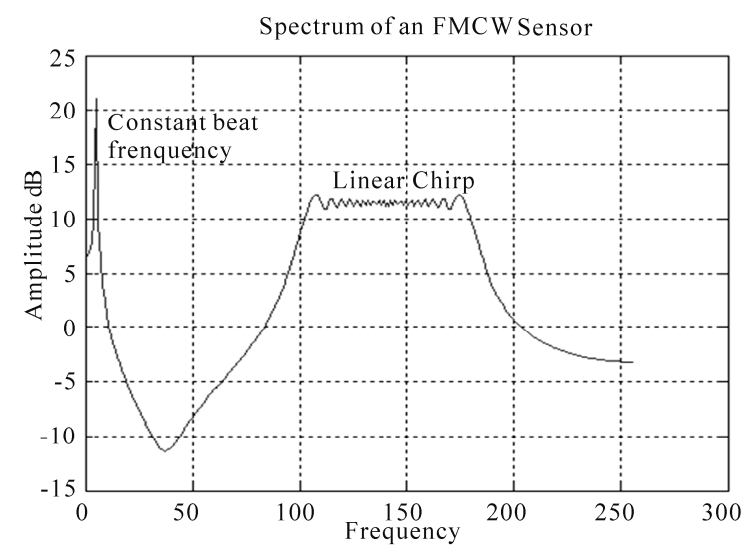

Figure 2. Frequency domain representation of FMCW sensor output

where $N_{L} \leq m \leq\left(N_{L}+N_{B}\right)$,

Now, we use Fourier Transformation, we get

$$
\begin{array}{r}
V_{F M C W 1}(f)=\frac{V_{i}^{2}}{2} \operatorname{Cos}\left(2 \pi f_{c} \tau_{f b}+\pi \frac{B}{T_{m}} \tau_{f b}^{2}\right) \\
\Delta T \int_{0}^{\infty} \operatorname{Cos}\left(2 \pi f_{o}((l-n) \Delta T)+\pi \frac{B}{T_{m}}((l-n) \Delta T)^{2}\right) \mathrm{h}(\mathrm{n} \Delta T) \mathrm{e}^{-\mathrm{j}(\mathrm{m} 2 \pi f \mathrm{n} \Delta \mathrm{T})} \Delta T
\end{array}
$$

And also we can get

$$
V_{F M C W 2}(f)=\frac{V_{i}^{2}}{2} \operatorname{Cos}\left(2 \pi f_{c} \tau_{f b}+\pi \frac{B}{T_{m}} \tau_{f b}^{2}\right)
$$

describes a beat signal at a fixed frequency $f_{b}=\frac{B}{T_{f}} \tau$.

It can be seen that the signal frequency is directly proportional to the time delay time $\tau$, and hence is directly proportional to the round trip time to the target.

\section{Conclusions}

This paper presents the time domain linear system analysis for FMCW radar response by performing the inverse Fourier transform over the frequency-domain scattered field which obtained by calculating the integral over the illuminated surface using the free space Green's function. Then we got the received FMCW signal and transmitted FMCW signal, the product detection is implemented to get the beat signal. The Fourier transform is used to find the beat frequency.

\section{REFERENCES}

[1] W. V. T. Rusch and P. D. Potter, "Analysis of reflector antenna,” Academic, New York, pp. 46-49, 1970.

[2] R. F. Harrington, "Time-harmonic electromagnetic fields," McGraw-Hill, New York, pp. 127, 1961.

[3] E. M. Kennaugh and R. L. Cosgriff, "The use of impulse 
response in electromagnetic scattering problems," IRE Natl. Conv. Rec., Part 1, pp. 72-77, 1958.

[4] S. Hatamzadeh-Varmazyar and M. Naser-Moghadasi, "An integral equation modeling of electromagnetic scattering from the surfaces of arbitrary resistance distribution," Progress In Electromagnetics Research B, Vol. 3, pp. 157-172, 2008.

[5] C. A. Valagiannopoulos, "Electromagnetic scattering from two eccentric Metamaterial cylinders with frequency-dependent permittivities differing slightly each other," Progress In Electromagnetics Research B, Vol. 3, pp. 23-34, 2008.

[6] E. M. Kennaugh and D. L. Moffatt, "Transient and impulse response approximation," Proceeding IEEE, pp. 893-901, August 1965.

[7] S. Hoshi, Y. Suga, Y. Kawamura, T. Takano, and S. Shimakura, "Development of an FMCW radar at $94 \mathrm{Ghz}$ for observations of cloud particles-antenna section," Proceeding of the Society of Atmospheric Electricity of Japan, No. 58, pp. 116, 2001.

[8] T. Takano, Y. Suga, K. Takei, Y. Kawamura, T. Takamura, and T. Nakajima, "Development of an cloud pro- filing FMCW radar at $94 \mathrm{Ghz}$, International Union of Radio Science," General Assembly, Session FP, No. 1786, 2002.

[9] E. Yuan and W. V. T. Rusch, "Time-domain physical optics," IEEE Transactions on Antenna and Propagation, Vol. 42, No. 1, January 1994.

[10] L.-X. Yang, D.-B. Ge, and B. Wei, "FDTD/TDPO hybrid approach for analysis of the EM scattering of combinative objects," Progress In Electromagnetics Research, PIER 76, pp. 275-284, 2007.

[11] G. M. Sami, "Time-domain analysis of a rectangular reflector," Submitted.

[12] S. Ishigami, H. Iida, and T. Iwasaki, "Measurements of complex antenna factor by the Near 3-antenna method," IEEE Transaction on Electromagnetic Compatibility, Vol. 38, No. 3, August, 1996.

[13] L. Li and C.- H. Liang, "Analysis of resonance and quality factor of antenna and scattering systems using complex frequency method combined with model-based parameter estimation," Progress In Electromagnetics Research, PIER 46, pp. 165-188, 2004. 